\title{
A Comparative Study on Electrosorption Behavior of Carbon Nanotubes Electrodes Fabricated via Different Methods
}

Guang Zhu*, Hongyan Wang, and Li Zhang*

Anhui Key Laboratory of Spin Electron and Nanomaterials, Suzhou University, Suzhou 234000, P. R. China;

\begin{abstract}
:
The carbon nanotubes (CNTs) electrodes were fabricated via electrophoretic deposition (EPD), press and screen printing methods, respectively. The electrochemical properties and electrosorption performance of the CNTs electrodes were tested, respectively. Inhere, screen printing, as a conventional method for fabricating supercapacitor electrodes, was used for fabricating the CDI electrodes for the first time. Such a comparison is reasonably envisaged not only to be used to further understanding the influence of fabrication method on the electrode performance, but also to form a fundamental basis for CDI applications.
\end{abstract}

Keywords: Capacitive deionization; Carbon nanotubes; Electrophoretic deposition method; Press method; Screen printing method

\footnotetext{
* Corresponding author. Tel.: +86 557 2871006; E-mail address: zhlisuzh@163.com

* Corresponding author. Tel.: +86 557 3681973; E-mail address: guangzhu@ahsztc.edu.cn
} 


\section{Introduction}

Capacitive deionization (CDI) is a promising deionization technology for removing ionic species from aqueous solutions without high power consumption and secondary pollution [1-7]. When wastewater was pumped to flow through two opposite electrodes with an electric field applied, the charged ions in the solution were forced to move towards the surface of electrodes with counter-charges. As a result, the contaminated solution was purified.

Generally, porous carbon materials have been reported to be widely used in CDI process due to their good conductivity, high surface area, and suitable pore size distribution [8-11]. Among various carbon species, carbon nanotubes (CNTs), with high ratio of surface area to volume and good chemical stability are regarded as a suitable candidate for CDI electrodes [12-14]. It is known that the electrosorption capacity of CNTs electrode strongly depends on its morphology and structure, which are related to the electrode fabrication method. By now several methods, such as electrophoretic deposition (EPD), press and screen printing, have been employed to deposite CNTs to the conductive substrate to fabricate the CNTs electrode [15-18]. Zou et al. [18] used press method to fabricate the ion-selective CNTs film as CDI electrodes. A higher salt removal efficiency than that of traditional CNTs electrode was achieved. The CNTs electrodes were fabricated via EPD method for CDI [16]. The electrodes exhibited a comparable electrosorption capacity in $\mathrm{NaCl}$ solution to other conventional carbon electrodes. Therefore, the electrode fabrication methods play an important role in the electrosorption performance of CNTs electrodes by influencing their morphology and structure.

In this work, we fabricated the CNTs electrodes using three different methods: EPD, press and screen printing, and their electrosorption performance in $\mathrm{NaCl}$ solution was investigated. Screen printing, as a conventional method for fabricating supercapacitor electrodes [19,20], was used for fabricating the CDI electrodes for the first time. Such a comparison is reasonably envisaged not only to be used to further understanding the influence of fabrication method on the electrode performance, but also to form a fundamental basis for CDI applications.

\section{Experimental}

\subsection{Materials}


Multi-walled CNTs were purchased from Nanotech Port Co. Ltd. (Shenzhen, China) with detailed specifications as follows: length $(1-2 \mu \mathrm{m})$, diameter $(10-20 \mathrm{~nm})$ and purity $(\geqslant 95 \%)$. Polyvinyl alcohol (PVA), ethyl cellulose (EC) and terpilenol were purchased from Sinopharm Chemical Reagent Co. Ltd. All the reagents were used without further purification.

\subsection{Fabrication}

The CNTs electrodes were prepared by EPD method [16]. In brief, CNTs were dispersed in a mixture solution of acetone and ethanol (volume ratio 1:1) and $10 \mathrm{mg} \mathrm{Al}\left(\mathrm{NO}_{3}\right)_{3} \cdot 9 \mathrm{H}_{2} \mathrm{O}$ was added into the suspension to increase the deposition rate and improve the adhesion of the powder particles to the substrate. The EPD was conducted by applying a constant DC voltage of $40 \mathrm{~V}$ for $30 \mathrm{~min}$. Then the as-deposited CNTs film electrode, named as sample 1, was dried in the air at $40^{\circ} \mathrm{C}$ for $2 \mathrm{~h}$.

The press method was described as follows: firstly, $2.7 \mathrm{~g}$ PVA was dissolved into $97.3 \mathrm{~g}$ deionized water under stirring at $90^{\circ} \mathrm{C}$ for $4 \mathrm{~h}$. Then the carbon slurry was prepared by mixing 800 mg CNTs and $100 \mathrm{mg}$ carbon black with $2.7 \mathrm{~g}$ PVA solution, and stirred in an agate bowl till the CNTs were homogeneously dispersed in the mixture. The carbon slurry was pressed on the graphite paper. Finally, the as-CNTs electrode, named as sample 2, was dried at $80^{\circ} \mathrm{C}$ for $2 \mathrm{~h}$ in an oven.

The CNTs electrodes were prepared by screen printing method [19]. In brief, $1 \mathrm{~g}$ EC was added into $20 \mathrm{~g}$ terpilenol, and dissolved under stirring at $80^{\circ} \mathrm{C}$. Then the carbon slurry was prepared by mixing $850 \mathrm{mg}$ CNTs and $100 \mathrm{mg}$ carbon black with $1.05 \mathrm{~g}$ EC solution, and stirred in an agate bowl till the CNTs were homogeneously dispersed in the mixture. The CNTs were screen-printed on the graphite paper and then heated at $100^{\circ} \mathrm{C}$ for $1 \mathrm{~h}$. The obtained CNTs electrode was named as sample 3 .

\subsection{Characterization of CNTs electrodes}

The surface morphology and structure of CNTs electrodes were examined by scanning electron microscopy (SEM, JEOL JSM-LV5610). The contact angles of water on the surface of the prepared electrodes were measured with the sessile drop method by a contact angle goniometer (JC2000D, Powereach) using digital microgaophs of deionized water droplets. The cyclic voltammetry (CV) was investigated by using Autolab PGSTAT 302N electrochemical workstation in a three-electrode mode, including a standard calomel electrode as reference electrode and a platinum foil as counter 
electrode. $1 \mathrm{M} \mathrm{KCl}$ solution was used as electrolyte. The specific capacitance can be obtained from $\mathrm{CV}$ curves using the following equation:

$$
C=\tilde{i} /(v \times m)
$$

where $\tilde{i}$ is the average current $(\mathrm{A}), \mathrm{v}$ is the scan rate $(\mathrm{V} \mathrm{s}-1)$ and $\mathrm{m}$ is the mass of electrodes $(\mathrm{g})$.

\subsection{Batch-mode electrosorption experiments}

The electrosorption performance of CNTs electrodes fabricated using different methods was investigated by batch-mode electrosorption experiments with a continuously recycling system [21]. In each experiment, the analytical pure sodium chloride $(\mathrm{NaCl})$ was employed as the target solution with a volume of $50 \mathrm{~mL}$ and the flow rate was $40 \mathrm{~mL} / \mathrm{min}$. A direct voltage of $1.2 \mathrm{~V}$ was applied on the opposite electrode. The intial conductivity of $\mathrm{NaCl}$ solution was $50 \mu \mathrm{S} / \mathrm{cm}$, and the atmosphere temperature was kept at $298 \mathrm{~K}$. The relationship between conductivity and concentration was obtained according to a calibration table made prior to the experiment [22]. The concentration variation was continuously monitored and measured at the outlet of the unit cell by using an ion conductivity meter.

In our experiment, the salt removal and electrosorption capacity are defined as follows:

$$
\eta=\frac{\Phi_{0}-\Phi}{\Phi_{0}} \times 100 \%
$$

where $\Phi_{0}$ is initial solution conductivity and $\Phi$ is the final solution conductivity.

$$
\Gamma=\frac{\left(C_{0}-C_{\mathrm{e}}\right) \times V}{m}
$$

where $\mathrm{C}_{0}$ is initial $\mathrm{NaCl}$ concentration $(\mathrm{mg} \mathrm{L}-1), \mathrm{Ce}$ is the final $\mathrm{NaCl}$ concentration $(\mathrm{mg} \mathrm{L}-1), \mathrm{V}$ is the volume of $\mathrm{NaCl}$ solution $(\mathrm{L})$ and $\mathrm{m}$ is the mass of electrodes $(\mathrm{g})$.

\section{Results and discussion}

Fig. 1 shows SEM images of CNTs electrodes fabricated via different methods. It can be observed that CNTs in all the samples entangle with each other and show a good network structure with many mesopores, which provides more tunnels for the entering of the solution and allows hydrated ions easily to move onto the surface of the film [23]. However, different from Fig. 1(a), there are some particles in Fig. 1(b) and (c), which should be carbon black or binders used in press and screen 
printing methods. These particles may block the pores of the CNTs porous network, which is not beneficial to the electrosorption.

Fig. 2 shows the photographic images of water droplets on the surface of CNTs electrodes fabricated via different methods. From Fig. 2(a) - (c), it can be seen that the contact angles of water droplet on the surface of samples 1,2 and 3 are $61^{\circ}, 0^{\circ}, 77^{\circ}$, respectively. The good wettability of sample 2 is due to the addition of hydrophilic PVA binder. While for sample 3 , the use of hydrophobic EC binder decreases its affinity to the water.

Raman spectra are used to investigate the structure of CNTs in samples 1,2 and 3, as shown in Fig. 3. Obviously, all samples exhibit two obvious peaks at about 1344 and $1590 \mathrm{~cm}^{-1}$, corresponding to D-band and G-band, respectively. The D-band is related to disorder and arises due to the breathing mode of k-point phonons of $\mathrm{A}_{1 \mathrm{~g}}$ symmetry while the G-band is associated with the conjugated structure of $\mathrm{sp}^{2}$ carbon domains [24]. The intensity ratio $\left(\mathrm{I}_{\mathrm{D}} / \mathrm{I}_{\mathrm{G}}\right)$ of $\mathrm{D}$ band to $\mathrm{G}$ band is often used as a measure of disordered or defective degree of carbon materials [25]. The $\mathrm{I}_{\mathrm{D}} / \mathrm{I}_{\mathrm{G}}$ values of sample 1 , sample 2 and sample 3 are $0.72,0.91$ and 1.04, respectively, indicating that the structures of CNTs have been destroyed by both the pressing and screen-printing methods.

$\mathrm{CV}$ measurement was conducted at a scan rate of $50 \mathrm{mV} / \mathrm{s}$ under the potential of -0.5 to $0.5 \mathrm{~V}$. Fig. 4 shows CV curves of the electrodes. The shapes of CV curves are close to rectangle, indicating that the electrosorption process is reversible, and no obvious peaks associated with the Faradic reaction can be observed in the chosen potential range, revealing that the capacitive response comes from the double electric layer formation due to the Coulombic interaction rather than the electrochemical oxidation/reduction reaction [26]. The specific capacitance (C in F/g) of samples 1 , 2 and 3 are $90.13,34.62$ and $14.91 \mathrm{~F} / \mathrm{g}$, respectively. This result indicates that the electrode fabricated via EPD method should have a higher electrosorption capacity than the electrodes fabricated via press and screen printing methods.

Fig. 5 shows conductivity transients in $\mathrm{NaCl}$ solution for different samples during batch-mode experiments. It can be observed that once the voltage is imposed, ions are driven onto the electrodes and the conductivity decreases and reaches equilibrium after about $30 \mathrm{~min}$. The final solution conductivities are 44.3, 23.2 and $31.7 \mu \mathrm{S} / \mathrm{cm}$ for samples 1,2 and 3, respectively. The salt removal (\%) and electrosorption capacity (mg/g) of samples 1, 2 and 3 are listed in Table 1 . Sample 1 has an 
electrosorption capacity of $0.99 \mathrm{mg} / \mathrm{g}$, which is much higher than those of samples 2 and 3 . However, its salt removal is lowest (only 12.7\%). This is because the fabrication of CNTs electrode fabricated via EPD method dose not require the use of binder, which is necessary in press and screen printing methods. These binders are conductive badly and can block the pores of CNTs film, which is not beneficial to the electrosorption. Therefore, sample 1 exihibits a highest electrosorption capacity. However, the drawback of the EPD method is that the only small amount of CNTs can be deposited on the substrate. Therefore, the total salt removal is low. In press method, the hyhrophilic PVA binder, which is diffcult to be used in screen printing method, can improve the wettability of the electrode and ehance the ion adsorption. Therefore, both the salt removal and electrosorption capacity of sample 2 are higher than those of sample 3.

\section{Conclusions}

In summary, the CNTs electrodes were fabricated via EPD, press and screen printing methods, and their electrosorption performance in $\mathrm{NaCl}$ solution was investigated. The results show that the fabrication methods play an important role in electrosorption performance of CNTs electrodes by influencing their morphology structure, mass and water wettability. The EPD method should be a promising method for fabricating CNTs electrode, if the problem in the mass loading of CNTs using this method can be improved in the future. Currently press method should be an effective method for fabricating CNTs electrodes.

\section{Acknowledgments}

This work is supported by the National Science Foundation of China (NSFC) (21271136), the Provincial Natural Science Foundation of Anhui (1508085ME104 and 1408085MB40), the Important Project of Anhui Provincial Education Department (KJ2015A250), the Key Program of Outstanding Youth Talents in Higher Education Institution (GXYQZD2016343), the Scientific Research Foundation for the Anhui Provincial Returned Overseas Chinese Scholars (Electrospun low dimensional $\mathrm{TiO}_{2}$ nano-structures and application in photovoltaic devices), the Program of Innovative Research Team of Anhui Provincial Education Department (Photoelectric information materials and new energy devices).

\section{References}


[1] J. Dykstra, R. Zhao, P. Biesheuvel, A. van der Wal, Water Res. 88 (2016) 358.

[2] M. Suss, S. Porada, X. Sun, P. Biesheuvel, J. Yoon, V. Presser, Energy Environ. Sci. 8 (2015) 2296.

[3] Y. Liu, T. Chen, T. Lu, Z. Sun, D.H. Chua, L. Pan, Electrochim. Acta 158 (2015) 403.

[4] X. Xu, Y. Liu, M. Wang, X. Yang, C. Zhu, T. Lu, R. Zhao, L. Pan, Electrochim. Acta 188 (2016) 406.

[5] X. Xu, L. Pan, Y. Liu, T. Lu, Z. Sun, D.H. Chua, Sci. Rep. 5 (2015) 8458.

[6] A. Omosebi, X. Gao, J. Rentschler, J. Landon, K. Liu, J. Colloid Inter. Sci. 446 (2015) 344.

[7] W. Shi, H. Li, X. Cao, Z.Y. Leong, J. Zhang, T. Chen, H. Zhang, H.Y. Yang, Sci. Rep. 6 (2016) 18966.

[8] X. Xu, L. Pan, Y. Liu, T. Lu, Z. Sun, J. Colloid Inter. Sci. 445 (2015) 143.

[9] Q. Dong, G. Wang, T. Wu, S. Peng, J. Qiu, J. Colloid Inter. Sci. 446 (2015) 387.

[10]K. Sharma, Y.-H. Kim, J. Gabitto, R. Mayes, S. Yiacoumi, H. Bilheux, L. Walker, S. Dai, C. Tsouris, Langmuir 31 (2015) 1038.

[11]X. Xu, Z. Sun, D.H. Chua, L. Pan, Sci. Rep. 5 (2015) 11225.

[12]H. Li, S. Liang, M. Gao, G. Li, J. Li, L. He, Water Sci. Tech. 71 (2015) 83.

[13]S. Chung, H. Kang, J.D. Ocon, J.K. Lee, J. Lee, Curr. Appl. Phys. 15 (2015) 1539.

[14]L. Yang, Z. Shi, W. Yang, Surf. Coat. Technol. 251 (2014) 122.

[15]R.C. Carvalho, A. Mandil, K.P. Prathish, A. Amine, C. Brett, Electroanal. 25 (2013) 903.

[16]C. Nie, L. Pan, H. Li, T. Chen, T. Lu, Z. Sun, J. Electroanal. Chem. 666 (2012) 85.

[17]Q. An, A.N. Rider, E.T. Thostenson, ACS Appl. Mater. Interfaces 5 (2013) 2022.

[18]J. Yang, L. Zou, N.R. Choudhury, Electrochim. Acta 91 (2013) 11.

[19]K. Jost, D. Stenger, C.R. Perez, J.K. McDonough, K. Lian, Y. Gogotsi, G. Dion, Energy Environ. Sci. 6 (2013) 2698.

[20]Y. Xu, M.G. Schwab, A.J. Strudwick, I. Hennig, X. Feng, Z. Wu, K. Müllen, Adv. Energy Mater. 3 (2013) 1035.

[21]G. Zhu, W. Wang, X. Li, J. Zhu, H. Wang, L. Zhang, RSC Adv. 6 (2016) 5817.

[22]H.B. Li, T. Lu, L.K. Pan, Y.P. Zhang, Z. Sun, J. Mater. Chem. 19 (2009) 6773. 
[23]X.Z. Wang, M.G. Li, Y.W. Chen, R.M. Cheng, S.M. Huang, L.K. Pan, Z. Sun, Appl. Phys. Lett. 89 (2006).

[24]W. Chaikittisilp, M. Hu, H. Wang, H.-S. Huang, T. Fujita, K.C.-W. Wu, L.-C. Chen, Y. Yamauchi, K. Ariga, Chem. Commun. 48 (2012) 7259.

[25]X. Wen, D. Zhang, T. Yan, J. Zhang, L. Shi, J. Mater. Chem. A 1 (2013) 12334.

[26]Z. Li, B. Song, Z. Wu, Z. Lin, Y. Yao, K.-S. Moon, C. Wong, Nano Energy 11 (2015) 711. 


\section{Figure Captions}

Fig. 1. Typical SEM images of (a) sample 1, (b) sample 2 and (c) sample 3.
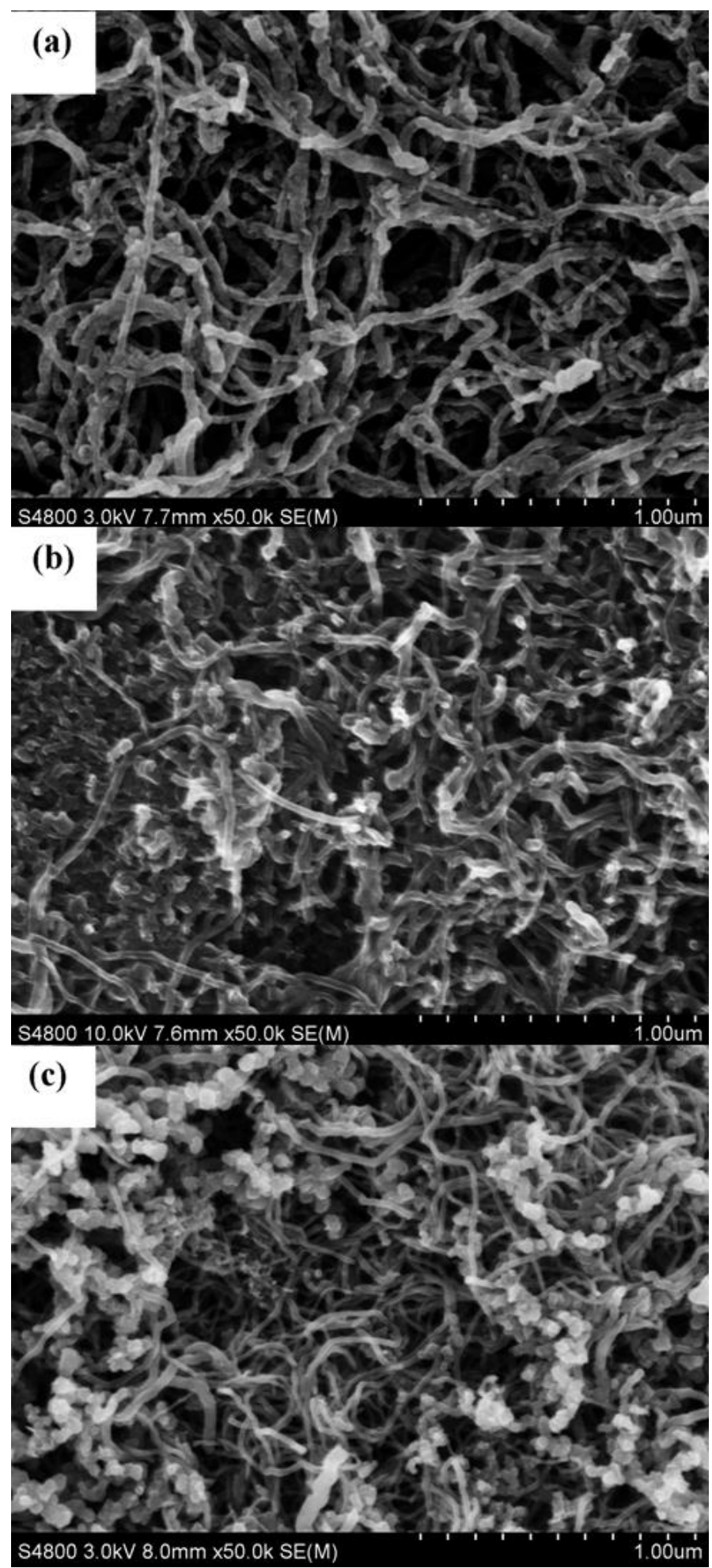
Fig. 2. Photographic images of water droplets on the surface of (a) sample 1, (b) sample 2 and (c) sample 3.

\section{(a)}

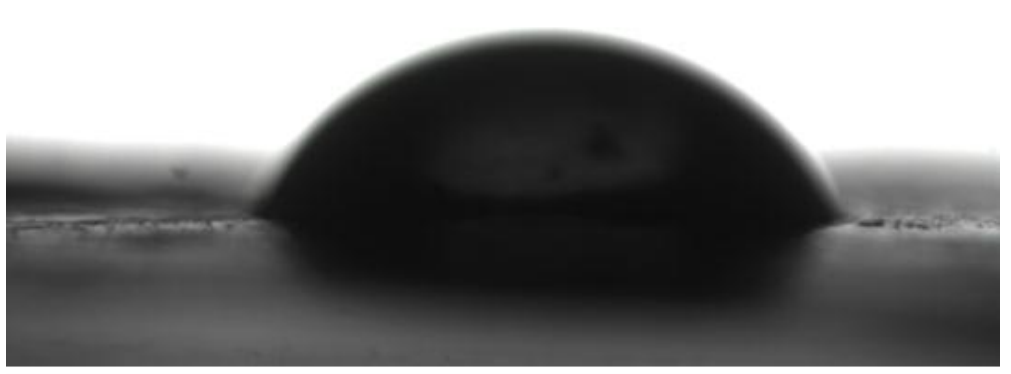

(b)

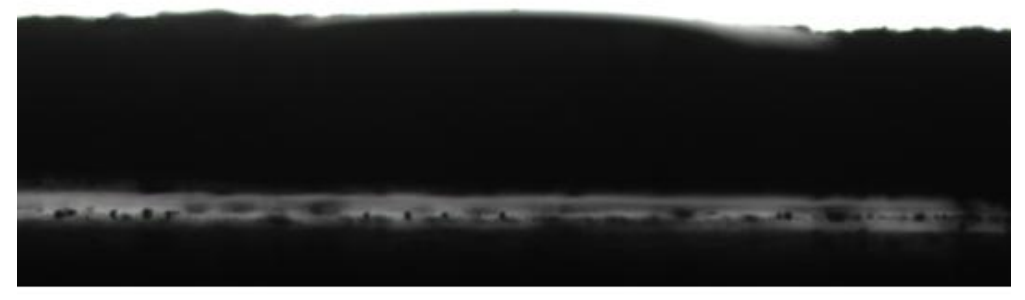

(c)

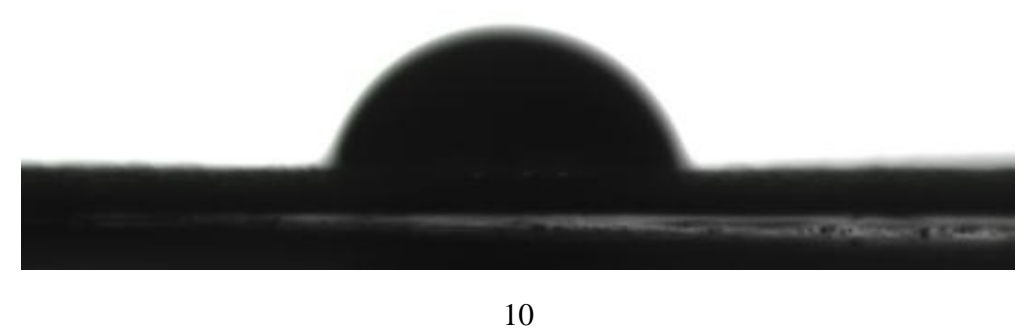


Fig. 3. Raman spectra of CNTs in samples 1, 2 and 3.

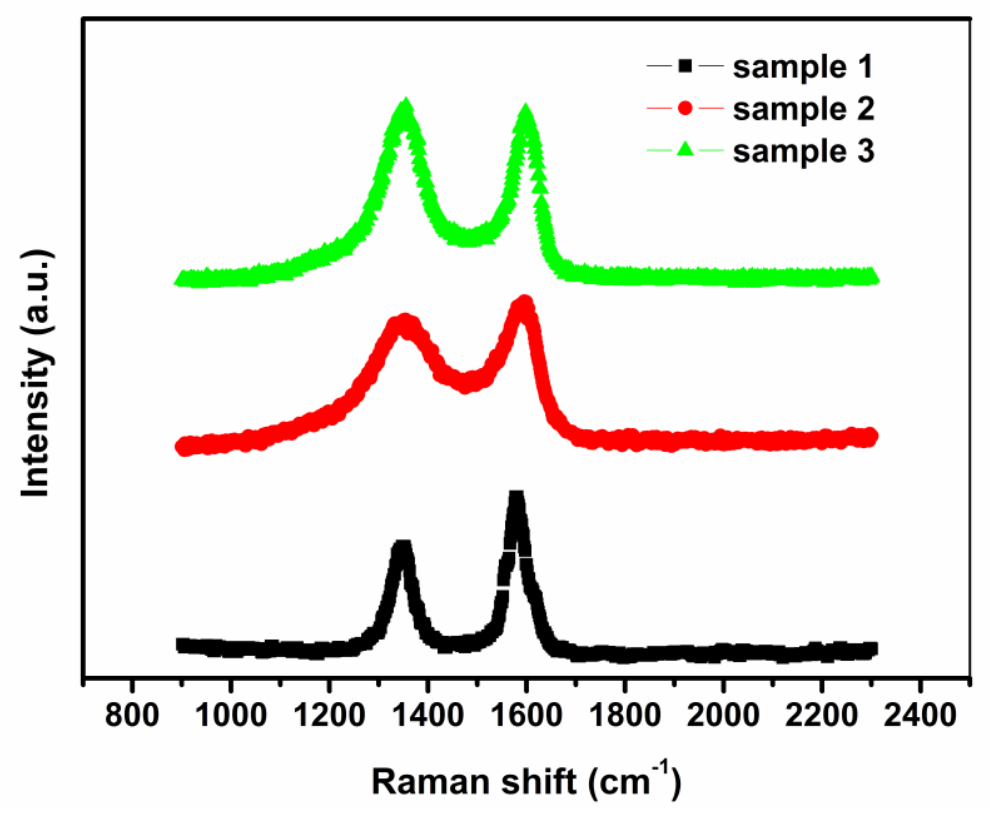

Fig. 4. $\mathrm{CV}$ curves of samples 1,2 and 3 in $1 \mathrm{M} \mathrm{KCl}$ solution at scan rate of $50 \mathrm{mV} / \mathrm{s}$.

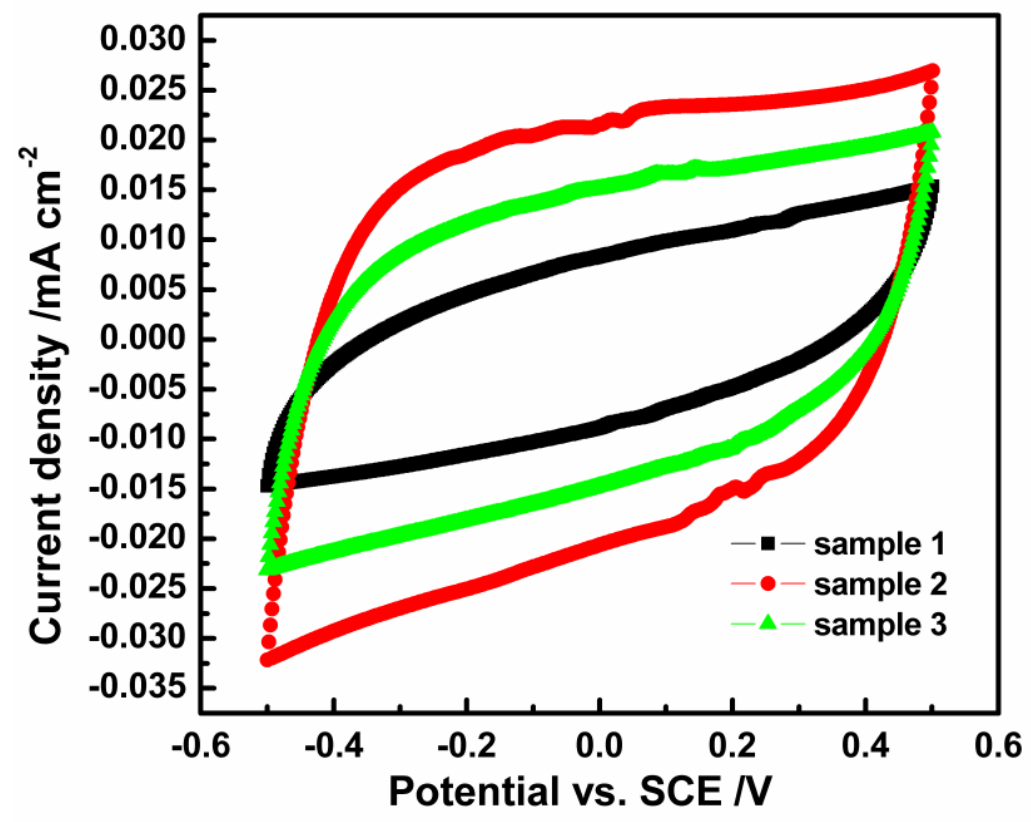


Fig. 5. Conductivity transients in $\mathrm{NaCl}$ solution for different samples during batch-mode experiments with an applied voltage of $1.2 \mathrm{~V}$.

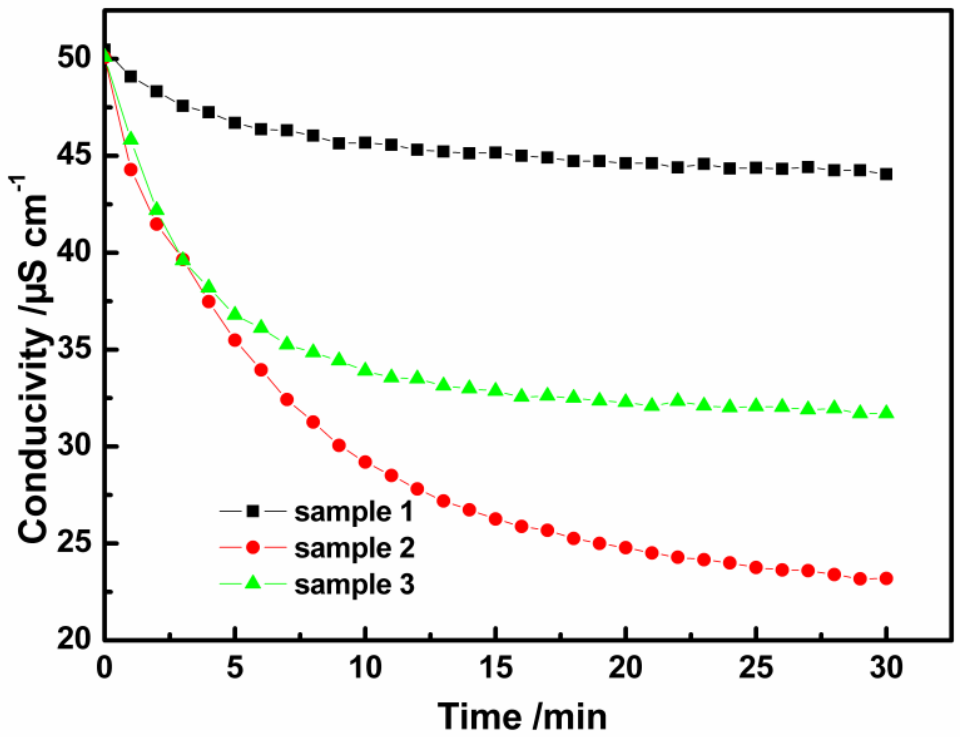

Table 1 Comparison of electrosorption capacity and salt removal among different samples.

\begin{tabular}{cccc}
\hline Sample & Mass of CNTs (g) & $\eta(\%)$ & $\Gamma(\mathrm{mg} / \mathrm{g})$ \\
\hline 1 & 0.161 & 12.7 & 0.99 \\
2 & 1.21 & 53.9 & 0.56 \\
3 & 1.40 & 36.7 & 0.33 \\
\hline
\end{tabular}




\section{Graphical Contents Entry}
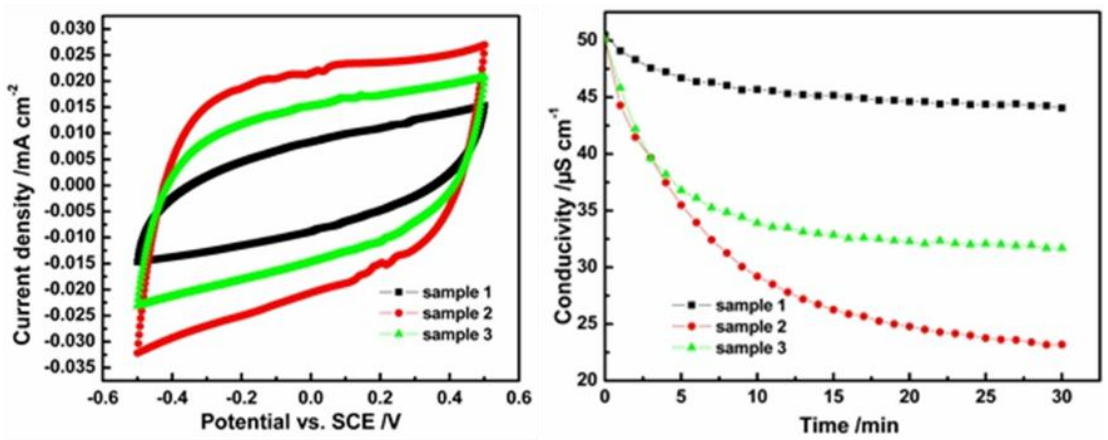

In this work, CNTs electrodes were prepared via using three different methods: EPD, press and screen printing, and their electrosorption performance in $\mathrm{NaCl}$ solution was investigated. The results show that the CNTs electrodes fabricated via EPD method exhibit a specific capacitance of $90.13 \mathrm{~F} / \mathrm{g}$, which is higher than that of the CNTs electrodes fabricated via press and screen printing methods. 